Bundesgesundheitsbl 2014 $\cdot 57: 1120-1126$ DOI 10.1007/s00103-014-2021-5

Online publiziert: 29. Juli 2014

c) Springer-Verlag Berlin Heidelberg 2014

R. Strobl ${ }^{1,2} \cdot$ W. Maier ${ }^{3} \cdot$ A. Mielck ${ }^{3}$ J. Fuchs ${ }^{4} \cdot$ A. Richter-Kornweitz ${ }^{5}$. J. Gostomzyk $k^{6} \cdot$ E. Grill ${ }^{1,2}$

${ }^{1}$ Institut für medizinische Informationsverarbeitung, Biometrie und Epidemiologie, Ludwig-Maximilians-Universität München, München

${ }^{2}$ Deutsches Schwindel- und Gleichgewichtszentrum (IFB), Klinikum der Universität München, München

${ }^{3}$ Institut für Gesundheitsökonomie und Management im Gesundheitswesen, Helmholtz Zentrum München, Neuherberg

${ }^{4}$ Abteilung Epidemiologie und Gesundheitsmonitoring, Robert Koch-Institut, Berlin

${ }^{5}$ Landesvereinigung für Gesundheit \& Akademie für Sozialmedizin Niedersachsen, Hannover

${ }^{6}$ Landeszentrale für Gesundheit in Bayern e. V., München

\title{
Wohnumfeld - Stolperstein oder Weg zum gesunden Altern?
}

\author{
Ergebnisse der Augsburger \\ Regionalkonferenz,,Wohnumfeld, \\ Alter und Gesundheit"
}

Die gestiegene Lebenserwartung und der damit verbundene demografische Wandel bringen nicht nur viele Herausforderungen für unsere Gesellschaft und unser Gesundheitssystem mit sich, sondern bergen auch eine Vielzahl an Chancen. Über den steigenden Ausgaben für die Gesundheitsversorgung und für das Rentenversicherungssystem sowie dem steigenden Bedarf an Pflege und Betreuung wird zu oft vergessen, dass ältere Menschen auch einen wichtigen Beitrag für die Gesellschaft leisten, sei es als versorgende und pflegende Familienmitglieder oder als ehrenamtlich tätige, aktive Teilnehmer ihrer Gemeinde. Sie stellen daher eine erhebliche soziale und wirtschaftliche Ressource dar. Auch aus diesem Grund gilt es, die Gesundheit und die soziale Teilhabe älterer Menschen zu fördern.

Neben einer guten medizinischen Versorgung ist hierfür die Struktur des Wohnumfeldes von zentraler Bedeutung: Sie kann selbstständiges Leben im Alter und ausreichende soziale Teilhabe sowie Kommunikation in der Gesellschaft fördern und damit einen wertvollen Beitrag für die individuelle Gesundheit im Alter leisten [1]. Für die sog. ,jungen Alten“ (bzw. „Best Ager") stellen die kommunale
Infrastruktur sowie soziale Netzwerke eine grundlegende Ressource für die Aufrechterhaltung der Unabhängigkeit und Selbstständigkeit dar [2]. Wer ein Wohnumfeld vorfindet, das im täglichen Leben unterstützend wirkt, der fühlt sich wohler und ist oft aktiver in der Durchführung von Alltagsaktivitäten [3]. Für die Kommunen bedeutet dies, dass sie der altersgerechten Gestaltung des direkten Wohnumfelds hohe Priorität einräumen und die bestehenden Strukturen fortlaufend kritisch überprüfen und optimieren sollten. Diese Ziele können jedoch nur erreicht werden, wenn die Politik auf Bundes-, Landes-, aber auch auf Gemeindeebene die Voraussetzungen für eine altersgerechte Gestaltung der Wohnumwelt schafft, d. h. einer Umwelt, die die speziellen Bedürfnisse älterer Menschen berücksichtigt, sie bei ihren alltäglichen Tätigkeiten unterstützt und den Zugang zu wichtigen Versorgungsstrukturen ermöglicht [4].

Die Probleme sind hierbei von Gemeinde zu Gemeinde unterschiedlich, daher müssen Programme aufgestellt werden, die auf diese Besonderheiten eingehen und vorhandene Potenziale aufdecken und integrieren. Unabdingbar ist da- bei die Kooperation mit den älteren Mitmenschen: Nur mit ihr ist es möglich, die spezifische Lebenssituation zu berücksichtigen und den individuellen Bedarf zu ermitteln. Wie sehen ältere Menschen ihre eigene, konkrete Situation in ihrem unmittelbaren Wohnumfeld und darüber hinaus? Auf was sollten die Kommunen achten, um „erfolgreiches Altern“ ihrer Bürgerinnen und Bürger fördern zu können?

Diesen Fragen sind wir im Rahmen der KORA-Age-Studie nachgegangen. Die Untersuchungen zur Bedeutung des Wohnumfelds für die soziale Teilhabe älterer Menschen basieren auf Interviews, Fokusgruppendiskussionen und der Verwendung eines Geoinformationssystems (GIS).

Die Ergebnisse unserer Untersuchungen wurden am 1. Oktober 2013 auf einer Regionalkonferenz im Augsburger Rathaus vorgestellt und dort mit politischen Entscheidungsträgern aus Augsburg und älteren Bürgern und Bürgerinnen aus der Region diskutiert. Zur Diskussion der Frage, wie Wissenschaft und Politik bei der Schaffung eines altersgerechten Wohnumfelds zusammenarbeiten können, wurden die Ergebnisse von natio- 
Tab. 1 Ausgewählte Meta-Codes, mit Sub-Codes und Zitate aus den Fokusgruppendiskussion

\begin{tabular}{|c|c|c|}
\hline Meta-Code & Sub-Code & Zitat \\
\hline \multirow[t]{3}{*}{ Aktivitäten der Bürger } & Kultur & „Das Singen brauch ich, die Menschen brauch ich" \\
\hline & Ehrenamt & $\begin{array}{l}\text { „... dann hab ich noch eine alte Dame, eine Nachbarin, die ist } 94 \text { und lebt noch zu Hause. } \\
\text { Und um die kümmere ich mich auch ..." }\end{array}$ \\
\hline & Familie & „... das sind eigentlich meine Hauptaktivitäten. Familie und Kinder" \\
\hline \multirow[t]{3}{*}{$\begin{array}{l}\text { Motivationsfaktoren zur } \\
\text { Teilhabe }\end{array}$} & Mobilität & $\begin{array}{l}\text { "....ich fahr auch noch Auto, fahre Fahrrad, bin also noch relativ mobil und dadurch habe ich } \\
\text { mit all dem keine Probleme“ }\end{array}$ \\
\hline & Innere Motivation & $\begin{array}{l}\text { „....ich musste keine Eltern pflegen, ich musste niemand pflegen, die Zeit stell ich einfach } \\
\text { zur Verfügung“ }\end{array}$ \\
\hline & Geselligkeit & $\begin{array}{l}\text { "Ich mach die ehrenamtlichen Tätigkeiten, dass ich Arbeit habe, das ich unter Menschen bin } \\
\text { und manchmal kommen da nette Gespräche zustande" }\end{array}$ \\
\hline Einfluss von Gesundheit & Gesundheit von Angehörigen & $\begin{array}{l}\text { ".... seit meine Frau ziemlich krank ist, muss ich viel im Haushalt machen. Da bleibt mir nicht } \\
\text { mehr viel Zeit, dass ich sonst was unternehme“ }\end{array}$ \\
\hline Sicherheit & Kriminalität & $\begin{array}{l}\text { "....keine Aktivitäten wollte, die alle abends stattfinden, wo ich dann nachts alleine nach } \\
\text { Hause müsste. So was wollte ich nicht" }\end{array}$ \\
\hline \multirow[t]{3}{*}{$\begin{array}{l}\text { Infrastruktur: negative } \\
\text { Aspekte }\end{array}$} & Öffentliche Verkehrsmittel & $\begin{array}{l}\text { „....ich steh halt } 20 \text { min da oben an der Haltestelle. Und so lustig ist die Haltestelle nicht da } \\
\text { oben“ }\end{array}$ \\
\hline & Anbindung & „Die Transportmöglichkeiten, wenn man so ein Ruf-Taxi hätte, dass wäre schön“ \\
\hline & Fahrrad & $\begin{array}{l}\text { "Wir müssen fast in der Mitte fahren, damit die Autofahrer nicht nervös werden und über- } \\
\text { holen wollen, weil das ist wirklich riskant" }\end{array}$ \\
\hline $\begin{array}{l}\text { Versorgung: negative } \\
\text { Aspekte }\end{array}$ & Einkaufen & „... jemand der nicht motorisiert ist, tut sich ganz schwer" \\
\hline \multirow{2}{*}{$\begin{array}{l}\text { Seniorenfreundlichkeit } \\
\text { des Wohnumfelds }\end{array}$} & Öffentliche Gebäude & „Ein öffentliches Klo im Zentrum gibt es nicht“ \\
\hline & Öffentliche Verkehrsmittel & $\begin{array}{l}\text { „Dann bin ich fast gezwungen nach Augsburg zu fahren und ... am Schalter mir die Fahr- } \\
\text { karten zu holen. Weil ich hab kein Internet und hab auch keinen Computer oder irgendwas } \\
\text { und kann auch mit so einem Zeug auch nicht umgehen. Auch mit diesen Automaten kann } \\
\text { ich nicht umgehen“ }\end{array}$ \\
\hline \multirow[t]{2}{*}{ Wichtigste Änderung } & Gemeinschaft & $\begin{array}{l}\text { ".... ich tät mir nur wünschen, dass das Miteinander von Jung und Alt, dass das besser in der } \\
\text { Zukunft funktioniert" }\end{array}$ \\
\hline & Bauliche Änderungen & $\begin{array}{l}\text { „... in unseren wunderschönen Park ... da müsste es ein Bankrondell geben, damit die } \\
\text { Bänke ein bisschen rund sind, damit man da auch miteinander reden kann“ }\end{array}$ \\
\hline
\end{tabular}

nalen Expertinnen und Experten aus verschiedenen Blickwinkeln kommentiert. Konkret ging es dabei auch um die Frage, welche stadtplanerischen Empfehlungen aus den Ergebnissen der Studie abgeleitet werden können.

Im Folgenden werden die inhaltlichen Schwerpunkte der einzelnen Beiträge kurz zusammengefasst und die wichtigsten Punkte der Diskussion erläutert.

\section{Die Perspektive der Bürger - Ergebnisse aus den Fokusgrup- pendiskussionen (Ralf Strobl)}

Die kommunale Infrastruktur, aber auch soziale Netzwerke stellen eine wichtige Ressource für die Aufrechterhaltung der Unabhängigkeit und Selbstständigkeit dar $[1,5]$. Diese Unabhängigkeit und Selbstständigkeit soll möglichst bis ins hohe Alter erhalten werden. Um gesund und erfolgreich altern zu können, ist die Schaffung von die Gesundheit fördernden Le- benswelten eine wesentliche Voraussetzung [6]. Dies kann jedoch nur erreicht werden, wenn die Politik auf Landesebene, aber auch auf kommunaler Ebene die Voraussetzungen für eine altersgerechte Umwelt schafft. Um aus erster Hand in Erfahrung zu bringen, welches die spezifischen Problemfelder, aber auch Potenziale in den Kommunen sind, wurden in Augsburg und den umliegenden Kommunen Bobingen und Gersthofen Fokusgruppendiskussionen durchgeführt [7].

Die Fokusgruppendiskussionen wurden mittels eines interdisziplinär erarbeiteten Interviewleitfadens teilstandardisiert durchgeführt und von zwei Moderatoren geleitet. Die Auswertung der transkribierten Gespräche erfolgte durch zwei Wissenschaftler mit der Methodik der qualitativen Inhaltsanalyse nach Mayring [8]. Die wichtigsten Themen wurden in sog. "Meta-Codes“ zusammengefasst. Diese Meta-Codes wurden dann, soweit erforderlich, in weitere Sub-Codes unterteilt.

Es wurden 11 Fokusgruppendiskussionen mit insgesamt 78 Teilnehmern durchgeführt. Aus den transkribierten Texten wurden 1230 Aussagen extrahiert und diesen 10 Meta-Codes und 34 spezifischeren Sub-Codes zugeordnet. Ausgewählte Meta-Codes mit den jeweiligen Sub-Codes und Zitaten sind in • Tab. 1 abgebildet.

In den Fokusgruppendiskussionen wurde Mobilität bzw. Erreichbarkeit von subjektiv wichtigen Orten als ein essenzieller Aspekt zur erfolgreichen und gewünschten sozialen Teilhabe genannt. Zum Beispiel haben ältere Menschen durch den Wegzug der Supermärkte hinaus auf die „grüne Wiese“, also an die Peripherie der Kommunen, Probleme ihre Einkäufe zu organisieren. Ein Auto oder ein Fahrrad fahren zu können ist für die eigene Mobilität oft unumgänglich. 
Die Erreichbarkeit der Innenstadt von Augsburg war ein wichtiges Diskussionsthema bei den Bewohnern der umliegenden Gemeinden, einerseits wegen der guten Einkaufsmöglichkeiten, andererseits wegen des kulturellen Angebots. Öffentliche Verkehrsmittel nach Augsburg haben daher eine besonders große Bedeutung. Die Wartezeiten, die Umsteigezeiten und die Gestaltung der Bahnhöfe wurden als nur bedingt seniorenfreundlich eingestuft. Die Teilnehmer nannten moderne Automaten und unbesetzte Service-Schalter als weitere Hindernisse für die eigene Mobilität und Selbstverwirklichung.

Die Teilnehmer waren insgesamt sehr motiviert, an der Gemeinschaft weiter teilzuhaben, sich selbst einzubringen und anderen zu helfen. Soziale Netzwerke wie Familie und Nachbarschaften spielen dabei eine ebenso wichtige Rolle für das Wohlbefinden wie das Ausüben eines Ehrenamts.

\section{Zusammenhang zwischen sozialer Teilhabe, kommunalen Ressourcen und der Gesundheit älterer Menschen in Augsburg (Werner Maier)}

Auch im Ruhestand im eigenen Wohnumfeld körperlich aktiv zu sein und am gemeinschaftlichen Leben teilhaben $\mathrm{zu}$ können (soziale Teilhabe) ist eine grundlegende Voraussetzung für gesundes Altern. Hierfür muss das Wohnumfeld eine entsprechende „soziale Infrastruktur“ aufweisen, d. h. gut zugängliche und in zumutbarer Entfernung liegende Dienstleistungen und Versorgungseinrichtungen sowie die Möglichkeit, bequem den öffentlichen Nahverkehr nutzen zu können. Gerade vor dem Hintergrund des demografischen Wandels ist es zunehmend wichtig, Zusammenhänge zwischen Wohnumfeld, sozialer Teilhabe und Gesundheit zu untersuchen.

Ziel dieser Studie war es, den Zusammenhang zwischen sozialer Teilhabe und Gesundheitszustand bei der älteren Bevölkerung in Augsburg und Umgebung zu untersuchen, und zwar mit Fokus auf a) die gewünschte und tatsächlich soziale Teilhabe und b) die objektiven und subjektiven Ressourcen im Wohnumfeld.

Bundesgesundheitsbl 2014·57:1120-1126 DOI 10.1007/s00103-014-2021-5

c) Springer-Verlag Berlin Heidelberg 2014

\section{R. Strobl · W. Maier · A. Mielck · J. Fuchs · A. Richter-Kornweitz · J. Gostomzyk · E. Grill \\ Wohnumfeld - Stolperstein oder Weg zum gesunden Altern? Ergebnisse der Augsburger Regionalkonferenz „Wohnumfeld, Alter und Gesundheit"}

\section{Zusammenfassung}

Neben einer guten medizinischen Versorgung ist die Struktur des Wohnumfeldes von zentraler Bedeutung, um soziale Teilhabe älterer Menschen zu fördern. Für die Kommunen bedeutet dies, dass sie der altersgerechten Gestaltung des direkten Wohnumfelds hohe Priorität einräumen sollten. Am 1. Oktober 2013 wurden auf der Regionalkonferenz „Wohnumfeld, Alter und Gesundheit "im Augsburger Rathaus verschiedene Ergebnisse der KORA-Age-Studie zum Thema Teilhabe und Wohnumfeld vorgestellt und mit politischen Entscheidungsträgern aus Augsburg und Seniorenvertretern aus der Region diskutiert. Die Studie untersuchte den Zusammenhang zwischen Wohnumfeld und Teilhabe mittels zweier unterschiedlicher Ansätze: qualitative Ergebnisse aus Fokusgruppendis- kussionen und quantitative Ergebnisse basierend auf Telefoninterviews und der Verwendung eines Geoinformationssystems. Die Ergebnisse wurden durch Beiträge aus regionaler und überregionaler Sicht ergänzt. In der Diskussion ergab sich, dass nur mit einem breiten Spektrum an Maßnahmen ein seniorenfreundliches Wohnumfeld geschaffen werden kann, sodass einerseits physische Barrieren abgebaut und andererseits das Gemeinschaftsgefühl, der nachbarschaftliche Zusammenhalt und das Miteinander gefördert werden.

\section{Schlüsselwörter}

Altersforschung · Teilhabe · Wohnumfeld . Regionalkonferenz · Gesundes Altern

\section{Living conditions: stumbling block or path to successful ageing? Results of the Augsburg regional conference "Living environment, age and health"}

\section{Abstract}

In addition to good medical care, living environment is of central importance in encouraging social participation among older people. Therefore, municipalities should prioritise the age-appropriate design of living environments. Results of the KORA Age study were presented at the regional conference "Living environment, age and health" in the Augsburg town hall on October 1, 2013. The results on participation and living environment were discussed with local policy makers and senior citizens' representatives from Augsburg and two surrounding regions. The study examined the impact of living environment on participation using two different approaches: qualitative findings from focus group discussions and quantitative find- ings based on telephone interviews and the use of a geographic information system. The results were complemented by contributions from a regional and national perspective. It was stressed in the closing discussion that a senior-friendly living environment can only be created by using a broad range of different measures. On the one hand physical barriers need to be removed, while at the same time the sense of community, neighborhood cohesion and solidarity should be encouraged further.

\section{Keywords}

Research on ageing $\cdot$ Participation . Living environment $\cdot$ Regional conference . Healthy ageing
Im Rahmen der KORA-Age Studie befragten wir 822 Männer und Frauen zwischen 68 und 96 Jahren. Über Telefon- und Untersuchungsinterviews sowie Selbstausfüllbögen wurden Angaben zu demografischen und sozioökonomischen Merkmalen, zur sozialen Teilhabe sowie zu subjektiven Ressourcen und Barrieren erfragt (z. B. Möglichkeiten der sozialen Teilhabe, subjektive Gehdistanzen zu wichtigen infrastrukturellen Einrichtungen, Bewertung des Wohnumfelds). Für die Stichprobe von Bewohnern der Stadt Augsburg wurden zudem die Geodaten sowohl von der (anonymisierten) Wohnadresse als auch von wichtigen infrastrukturellen Adressen (z. B. Hausärzte, Apotheken) in ein Geographisches Informationssystem (GIS) eingepflegt. Anschließend wurden die räumlichen Distanzen 
über eine Netzwerkanalyse berechnet. Diese objektiven Distanzen sollen später mit den erfragten subjektiven Distanzen der Probanden verglichen werden.

Erste Analysen zeigten, dass von den Personen, welche ihre Wohnung gerne häufiger verlassen würden, rund $40 \%$ physische Barrieren (z. B. Treppen oder Gehsteige) und rund $60 \%$ einen eingeschränkten Gesundheitszustand als wichtigsten Hinderungsgrund angaben. Die fußläufige Erreichbarkeit von Versorgungseinrichtungen und Dienstleistungen zeigte ein heterogenes Bild: während z. B. Lebensmittelgeschäfte bzw. Supermärkte oder Apotheken relativ gut erreichbar waren (bis zu 10 min: zwischen 45 und $50 \%$ der Befragten), konnte nur rund ein Drittel der Befragten einen Hausarzt innerhalb von 10 min zu Fuß erreichen, und für etwas mehr als ein Drittel der Befragten war der Hausarzt sogar mehr als 20 min entfernt. Ein ganz ähnliches Bild ergab sich auch bei der objektiven Berechnung der räumlichen Distanzen. Angebote für seniorenspezifische Beratung bzw. für Seniorentreffen kannten nur relativ wenige der Befragten, und diese Angebote wurden auch nur relativ wenig genutzt. Eingeschränkte Möglichkeiten der sozialen Teilhabe zeigten sich vor allem bei Frauen, bei älteren Personen und bei Personen mit niedriger Bildung bzw. niedrigem Einkommen.

\section{Wohnumfeld und Gesundheit - Diskussionsbeitrag aus Perspektive der Landesvereinigung für Gesundheit und Akademie für Sozialmedizin Niedersachsen (Antje Richter-Kornweitz)}

Viele Ältere, so auch die Mehrzahl der 60bis 79-Jährigen, bezeichnen den eigenen Gesundheitszustand als „gut" bis „sehr gut“. Einschränkungen bestehen jedoch je nach sozialer Lage oder auch nach Geschlecht. Arbeits- und Lebensbedingungen haben die Gesundheit über den Lebensverlauf geprägt, und die daraus resultierende materielle Situation beeinflusst sie im Alter. Dies prägt die Chancen auf soziale Teilhabe mit wechselseitiger Wirkung auf Gesundheitsstatus und die Chancen einer selbstbestimmten Lebensführung.

Einflussreich ist in dieser Lebensphase die Gestaltung des Wohnumfeldes, denn Ältere haben einen geringeren Radius für ihre Alltagsgeschäfte, und die dortigen Bedingungen entscheiden, wie autonom sie diese erledigen können. Daneben können positive Impulse zur Erhaltung eines selbstbestimmten Alltags von einem Beziehungsgeflecht aus unterstützenden sozialen Netzwerken und institutionellen Angeboten im Wohnumfeld ausgehen. Diese können tragfähige Strukturen bilden und so fördernde Nachbarschaften entstehen lassen. Befürworter einer solchen Strukturbildung im Wohnumfeld bezeichnen dies als „Speicherort für soziales Kapital“" [9] und betonen das gesundheitsfördernde Potenzial von gemeinsam verfolgten Zielen, vertrauensvollem Umgang und sozialem Zusammenhalt. Neben einer kleinräumigen Strukturbildung, zugeschnitten auf die Bedürfnisse der älteren Frauen und Männer sowie auf den lokalen, fachlich begründeten Bedarf, ist das „Stiften von Beziehungen“ erforderlich, um die Bedingungen in einem Wohnumfeld gesundheitsförderlich zu gestalten. Eine Strukturbildung wird unterstützt durch politischen Willen, sorgfältig ausgearbeitete Konzepte, die genaue Kenntnis der lokalen Strukturen und Akteure, die Entschlossenheit, auch geringste Handlungs- und Gestaltungsspielräume zu nutzen, und meist auch durch persönliches Engagement Einzelner.

Instrumentarium und Methodik für diesen Prozess bestehen in der Implementierung von leicht erreichbaren, gut sichtbaren Anlaufstellen im Wohnumfeld, differenzsensiblen Zugangsweisen und sektorenübergreifender Vernetzung und Kooperation. Das Stiften von Beziehungen erfordert zuallererst eine spezifische innere Haltung im Kontakt mit dem Gegenüber, die geprägt ist von Respekt und Wertschätzung: Dabei geht es auch darum, nicht zu viel und trotzdem genug zu tun, d. h. einerseits initiativ zu wirken und andererseits kontinuierlich auf vorhandene Ressourcen, Verselbstständigung und Selbstorganisation der Aktivitäten hin zu orientieren. Diese situationsangemessene Zurückhaltung und wert- schätzende Haltung erfordern eine hohe Aufmerksamkeit für den Prozess.

\section{Wohnen in Augsburg - Diskussionsbeitrag aus der Perspektive der Landeszentrale für Gesundheit in Bayern e.V. (Johannes Gostomzyk)}

Die Lebenserwartung der 65-Jährigen in Deutschland stieg zwischen 1900-1910 und 2011 für Männer von 10,4 auf 17,7 Jahre und von 11,9 auf 20 Jahre für Frauen. Der erfreuliche und im Trend anhaltende Gewinn kann als ein Angebot zur permanenten individuellen und lebensweltlichen gesundheitsbezogenen Prävention aufgefasst werden. Damit steigt auch die Chance, die gewonnene Lebenszeit möglichst unbelastet durch Krankheit, Behinderung und Abhängigkeit von Hilfeleistungen zu verbringen.

Altern ist ein biologischer und sozialer Prozess. Mit dem Alter steigt das Risiko chronischer Erkrankung, insbesondere von Herz und Kreislauf, von Psyche und Nervensystem sowie für Krebs. Präventives Verhalten und Aktivitäten der Gesundheitsförderung im Quartier sind keine Garantie für Krankheitsvermeidung und können das Altern nicht aufheben. Gleichwohl ist durch sie die Hoffnung berechtigt auf bessere Lebensqualität und auf Vermeidung oder zumindest Kompression von Krankheitszeiten. Wesentlicher Teil der Lebenswelt des Einzelnen ist sein Wohnquartier, also sein Stadtteil bzw. seine Kommune. Befragungen ergeben, ältere Menschen wünschen, eine möglichst lange Zeit in selbstbestimmter Lebensführung unter Beibehaltung bestehender sozialer Einbindung im gewohnten Stadtteil zu verbringen und hier bei Bedarf Unterstützung und Pflegeleistungen zu erhalten.

Die Stadtteilverbundenheit ist für das Wohlbefinden im Alter wichtig. Ältere Menschen gehören zur Stadt, und ihr Stadtteil gehört ihnen. Das entspricht dem Quartierskonzept der sozialen Stadt. Dieses zielt auflebenslanges Wohnen in Vierteln mit Versorgungssicherheit. Das Konzept basiert auf der sozialplanerischen Perspektive der Verknüpfung von Wohnungswirtschaft mit Anbietern von sozialen und medizinischen Dienstleistungen. 
Älterwerden mit gewachsenen sozialen Beziehungen (Familie, Nachbarn, Freunde) im gewohnten Quartier war im Wandel der Zeiten und bei sozialer Ungleichheit nicht für jedermann erreichbar. Es war offensichtlich auch nicht Leitbild kommunaler Altersfürsorge. In der ehemals Freien Reichsstadt Augsburg wurden zur Pflege bedürftiger Bürger zwischen 1150 und 19605 große, bis heute betriebene Stiftungshäuser errichtet. Sie wurden außerhalb der Stadtmauern erbaut und bedeuteten für die Bewohner räumliche Trennung von ihrer bisherigen Lebenswelt. Das früher typische Altenheim karitativer Fürsorge für in Not geratene Bürger existiert nicht mehr. Heute, im Zeitalter der Sozialversicherungen, gibt es über die Stadt verteilte 28 Senioreneinrichtungen mit 3065 vollstationären Plätzen. Das stationäre Angebot umfasst zeitgemäß betreutes Wohnen, Wohnheimpflege und Kurzzeitpflege. Auf 1000 Einwohner über 65 Jahre kommen 55 Pflegeplätze, der bayerische Durchschnitt liegt bei 45 Plätzen. Träger der Einrichtungen sind der städtische Eigenbetrieb Altenhilfe, die Freie Wohlfahrtspflege und seit Einführung der Gesetzlichen Pflegeversicherung (SGB XI) 1995 zunehmend auch private Anbieter („Seniorenresidenzen" u. Ä.). Daneben haben sich 43 Einrichtungen mit ambulanten Pflegediensten und auch bürgerschaftliches Engagement (Patenschaften u. a.) etabliert. Etwa $20 \%$ (ca. 53.000 Einwohner) der Stadt Augsburg (266.000 Einwohner) sind älter als 65 Jahre. Rund 3\% (ca. 8000 Einwohner) sind pflegebedürftig, davon werden ca. $70 \%$ zu Hause versorgt und 30\% in Einrichtungen. Die Zahl der Menschen mit Demenzerkrankungen wird auf 4000 geschätzt.

Die Landeszentrale für Gesundheit in Bayern (LZG) hat im Rahmen des Bayerischen Gesundheitsförderungs- und Präventionspreises (BGPP) im Jahr 2011 die Stadt Rödenbach ausgezeichnet für ihr Konzept: „Wie eine ganze Stadt daran arbeitet, dass ihre Senioren so lange wie möglich zu Hause bleiben können - Prävention und Versorgung für Hochbetagte in der Stadt". Partizipatives Element für die integrierte Stadt- und Entwicklungsplanung waren wiederholte Befragungen von Senioren seit 2002. Diese wünschten besonders eine barrierefreie Gestaltung des öffentlichen Raumes und ein differenziertes, an individuellen Bedarfen orientiertes Angebot „Häuslicher Hilfen “ für Hochbetagte. Das überfordert den Auftrag und die Ressourcen kommunaler Daseinsvorsorge. Der Schlüssel für das Gelingen des Konzeptes ist bürgerschaftliches Engagement. Die freiwillig und ehrenamtlich tätigen Bürger mit der Vision, etwas Bedeutsames zu tun, werden eingewiesen, begleitet und bekommen Anerkennung. Jüngere Senioren erkennen, dass ihre Arbeit in der Kommune auch für sie Hilfe in der Zukunft sichert.

In den kommenden Jahren wird der Anteil älterer Menschen in der Bevölkerung steigen. Mietpreissteigerungen in Innenstädten, negative Rentenentwicklungen und Altersarmut sind bereits reale Herausforderungen für eine soziale Seniorenpolitik, die auch Aktivitäten zur Gesundheitsförderung und zu gesundheitsbezogener Prävention beinhalten muss.

\section{Gesundheitsmonitoring - Diskussionsbeitrag aus Perspektive des Robert Koch-Instituts (Judith Fuchs)}

In den kommenden Jahren wird der Anteil der über 60-Jährigen an der bundesdeutschen Bevölkerung von $26 \%$ im Jahr 2010 auf geschätzte $40 \%$ im Jahr 2060 ansteigen [10] und damit die Gesundheit Älterer immer relevanter werden. Bei der Analyse des Gesundheitszustandes älterer und alter Menschen sollte, darauf weist die WHO hin, über das Erfassen von Erkrankungen hinaus eine Reihe von weiteren Faktoren, die diesen direkt oder indirekt beeinflussen, erfasst werden [11]. Das Wohnumfeld spielt hierbei eine wichtige Rolle.

Das Robert Koch-Institut (RKI), ein Bundesinstitut im Geschäftsbereich des Bundesministeriums für Gesundheit, hat als Kernaufgaben die Bekämpfung von Infektionskrankheiten und die Analyse gesundheitlicher Trends in der Bevölkerung. Die Aufgabenfelder des RKIs umfassen: das frühzeitige Erkennen von gesundheitsrelevanten Problemen in der Allgemeinbevölkerung und in Risikogruppen, das unabhängige Bewerten von Entwicklungen, Problemlagen und Risiken auf der
Basis von erhobenen Daten und das Entwickeln von Maßnahmen zur Gesundheitsförderung und Krankheitsverhütung (Handeln) [12]. Im Rahmen des Gesundheitsmonitorings des RKIs werden auch Daten zum Gesundheitszustand der älteren Bevölkerung erhoben. Damit wird das Erkennen und Bewerten von gesundheitsrelevanten Problemen Älterer auf Bundesebene ermöglicht.

Bei der Analyse des Gesundheitszustands älterer und alter Menschen sollten Umwelt- und Lebensbedingungen Berücksichtigung finden, so $\mathrm{z}$. B.

1. die Umgebung: mobilitätsfördernde und -einschränkende Faktoren (Fahrstühle, Rollator- und Rollstuhl-geeignete Wege, Barrieren), die Sicherheit (Beleuchtung, Belebtheit, Kriminalität), die Angebote des öffentlichen Personennahverkehrs ÖPNV, die Infrastruktur im Nahbereich (Lebensmittel, Banken, Post, Ärzte, Apotheken, Restaurants, Cafés, Theater, Buchläden, Kino) oder Grünflächen;

2. das pflegerische Versorgungsangebot: Pflegestützpunkte, Pflegedienste und Angebote hauswirtschaftlicher Unterstützung;

3. Angebote zur Teilhabe und Mobilitätsförderung: preiswerte Mittagstische, Mobilitätshilfedienste oder zielgruppenangepasste Freizeitangebote;

4. besondere Ressourcen zur Kompensation sozialer Benachteiligung: Förderung von soziokulturellen Zentren, Stadtteilzentren, Nachbarschaftsnetzwerken, Familien oder auch Wohnungsbaugenossenschaften;

5. lokale Steuerung und Vernetzung von Pflege und Seniorenarbeit in der Altenhilfeplanung, bei Seniorenbeiräten und Koordinierungsstellen.

Viele der genannten Aspekte (z. B. Grünflächen, Infrastruktur) sind für Menschen aller Altersgruppen wichtig, erlangen aber gerade im Alter besondere Bedeutung. So können Beschränkungen dieser Ressourcen dazu führen, dass das vertraute Umfeld verlassen werden muss, was negative Auswirkungen auf den Gesundheitszustand und das Wohlbefinden älterer Menschen haben kann. Im Sinne der Verhältnisprävention ist geplant, zukünftig Umwelt- und Lebensbedingungen noch bes- 
ser im Gesundheitsmonitoring des RKIs abzubilden, dabei sind die Erfahrungen und Ergebnisse aus der KORA-Age-Studie von großem Nutzen.

Bedürfnisse, Bedarfe und Fragen der Inanspruchnahme können sowohl nach dem Top-down-Prinzip vonseiten der Politik und Verwaltung der Städte/Regionen als auch nach dem Bottom-up-Prinzip durch Bürgerinnen und Bürger, Seniorenvertretungen oder Einrichtungen der Altenhilfe sowie vonseiten der Gesundheitsversorgung (Ärztinnen und Ärzte, Krankenhäuser, Pflegeeinrichtungen) thematisiert werden.

Die präsentierten Ergebnisse aus KORA-Age tragen in hohem Maße dazu bei, dass für die Region Augsburg Informationen zur Verfügung stehen, die in dieser Form bisher noch nicht zusammengestellt wurden: Sie unterstützen hierdurch das Erkennen und Bewerten von möglichen Problemlagen im Bereich des Wohnumfelds Älterer. Es ist zu hoffen, dass dies zum Handeln im Sinne der Initiierung von Maßnahmen führt, die die Lage Älterer und Alter nicht nur im Raum Augsburg verbessern.

\section{Diskussion und Fazit}

Diskussion und Fazit orientieren sich an den folgenden 5 Fragen.

\section{Wer hat an der Tagung teilgenommen?}

Neben der Vorstellung von Ergebnissen aus der KORA-Age-Studie zum Thema "Teilhabe" bestand ein wesentliches Ziel der Tagung darin, einen Personenkreis aus Vertretern der kommunalen Entscheidungsträger, aus nationalen Experten und Seniorenvertretern der beteiligten Kommunen zu einer offenen Diskussion zu bewegen. Insgesamt folgten ca. 40 Personen unserer Einladung. Aus der kommunalen Verwaltung der Stadt Augsburg waren Vertreter des Gesundheitsamts, des Amts für Soziale Leistungen, Senioren und Menschen mit Behinderung sowie des Sozialreferats und des Wohnung- und Stiftungsamtes anwesend. Als Interessenvertreter der Senioren kamen Personen aus den beteiligten Seniorenbeiräten und Vertreter von regi- onalen Anbietern für Seniorentreffpunkte wie der Altenhilfe oder dem evangelischem Forum Annahof in Augsburg. Als regionale Experten nahmen Wissenschaftler vom Helmholtz Zentrum München, der Ludwig-Maximilians-Universität München und der Universität Augsburg teil. Die kommunale Sichtweise von politischen Entscheidungsträgern wurde von Herrn Prof. Dr. Johannes Gostomzyk von der Landeszentrale für Gesundheit in Bayern und von Herrn Klaus Kneißl vom Amt für Soziale Leistungen, Senioren und Menschen mit Behinderung in Augsburg vorgestellt. Frau Dr. Antje Richter-Kornweitz von der Landesvereinigung für Gesundheit in Hannover und Frau Dr. Judith Fuchs vom Robert Koch-Institut in Berlin vertraten die überregionale Expertise.

\section{Welche Erwartungen hatten die Teilnehmer?}

Die Erwartungen an die Ergebnisse der Regionalkonferenz waren sehr unterschiedlich. Wunsch der Seniorenvertreter war es, mehr über konkrete Pläne und Strategien zur Verbesserung ihres direkten Wohnumfelds zu erfahren, während die Vertreter der Wissenschaft ihr Augenmerk auf den Wissensaustausch legten. Der direkte Austausch zwischen Wissenschaftlern, Vertretern der Stadt und Vertretern der Senioren fand nicht immer so intensiv statt wie gewünscht.

\section{Welche Probleme wurden bei der praktischen Umsetzung dieser Vorschläge diskutiert?}

Einer der zentralen Punkte in der Diskussion war die Frage, warum die Umsetzung von wissenschaftlichen Erkenntnissen in die Praxis so langwierig ist. So wurde in der Diskussion deutlich darauf hingewiesen, dass bei der Umsetzung der städtebaulichen Planung (z. B. in Bezug auf Barrierefreiheit) kein Erkenntnisdefizit, sondern ein Handlungsdefizit vorliegt. Kommunale Vertreter der Gemeinde nahmen dazu Stellung und erläuterten die Gründe, die dazu führen, dass notwendige Maßnahmen erst mit erheblicher Verzögerung umgesetzt werden. Insgesamt konzentrierte sich die Diskussion im Wesentlichen auf die erforderlichen Schritte bei der Implementierung von städtebaulichen oder strukturverändernden Maßnahmen, die für die Gestaltung einer seniorenfreundlichen bebauten Umwelt erforderlich sind. Hierbei gilt es, zwischen den Bedürfnissen und dem Bedarf der Bürgerinnen und Bürger zu unterscheiden, also den subjektiven Wünschen einerseits und den objektiv tatsächlich nötigen Verbesserungen andererseits. Während der strukturelle Bedarf weitestgehend bekannt ist, sind es die Bedürfnisse oft noch nicht.

Die Politiker und Stadtplaner erklärten sich bereit, hierfür enger mit der Wissenschaft zusammenzuarbeiten, wiesen allerdings auf die typischen Probleme bei der Umsetzung einer derartigen Zusammenarbeit hin: fehlende finanzielle Mittel sowie mangelnde Kooperationsbereitschaft lokaler Stellen. Weiterhin existiert eine augenscheinliche Diskrepanz zwischen gewünschten Maßnahmen und der späteren tatsächlichen Nutzung einer seniorenspezifischen Infrastruktur. Zum Beispiel werden Seniorenberatungsstellen nur von einem relativ kleinen Teil der älteren Bevölkerung genutzt, während viele ältere Menschen (insbesondere solche, die vergleichsweise bedürftiger erscheinen) solchen Angeboten oft fernbleiben.

\section{Welche konkreten Vorschläge wurden für Augsburg diskutiert?}

Nicht allein bauliche Änderungen sind entscheidend für ein seniorengerechtes Wohnumfeld. Vielmehr kann die erfolgreiche seniorenfreundliche Gestaltung des Umfelds nur über die Förderung des Miteinanders funktionieren. Dies ist eine gesamtgesellschaftliche Herausforderung, die weit über die lokalpolitische oder städteplanerische Ebene hinausgeht. Die aktuelle demografische Entwicklung erfordert es, wirksame Konzepte mit Einbeziehung von sozialen Netzwerken und nachbarschaftlichen Anstrengungen zu erstellen. Fortdauernde und tragfähige soziale Strukturen können sich jedoch ohne Unterstützung von außen (vor allem in Form von Ressourcen) nicht genügend ausbilden. Gerade in sozial benachteiligten Gebieten ist somit die Förderung von permanenten sozialen Strukturen durch externe Ressourcen (z. B. Personalstellen) unabdingbar. Frau Dr. Richter-Kornweitz for- 
mulierte diese Forderung als „Bauchtanz statt Blumenkübel“, also der vorrangigen Schaffung von Stellen und Programmen, die das Miteinander fördern (und der nachgeordneten Wichtigkeit von baulichen Maßnahmen).

\section{Was ist unser Fazit aus dieser Tagung?}

Nur mit einem breiten Spektrum an Maßnahmen kann es gelingen, ein seniorenfreundliches Wohnumfeld zu schaffen. So müssen einerseits physische Barrieren abgebaut und andererseits Programme zur Förderung des Miteinanders initialisiert werden. Bei der Planung dieser Maßnahmen müssen die älteren Mitbürger von Anfang an involviert sein, um Aktionen zu vermeiden, die am Bedarf und am Bedürfnis vorbeigehen. Programme, die auf kommunaler Ebene auf die Perspektive älterer Menschen eingehen, die vorhandenen Potenziale aufdecken und nutzen, sind unbedingt erforderlich. Wichtig sind hier vor allem Stellen, die soziale Netzwerke kreieren und pflegen, z. B. Kirchen oder Vereine. Diese gilt es zu unterstützen und zu stärken. Nur mit gemeinsamen Anstrengungen kann ein seniorengerechtes Wohnumfeld geschaffen und fortlaufend verbessert werden. Nicht zuletzt ist ein altersgerechtes Wohnumfeld auch für alle anderen Bewohner förderlich, z. B. für jüngere Familien.

\section{Korrespondenzadresse}

\section{Dr. R. Strobl}

Institut für medizinische

Informationsverarbeitung

Biometrie und Epidemiologie

Ludwig-Maximilians-Universität München,

Marchioninistr. 17, 81377 München

ralf.strobl@med.uni-muenchen.de

Danksagungen. Wir danken den beteiligten Kommunen in der KORA-Region Augsburg für die Unterstützung bei der Gestaltung und der Organisation der Konferenz sowie den Vertretern der jeweiligen Seniorenbeiräte für deren wertvolle Informationen und die große Unterstützung bei der Durchführung der Fokusgruppendiskussionen.

Die Forschungsplattform KORA (Kooperative Gesundheitsforschung in der Region Augsburg) wurde initiiert und finanziert vom Helmholtz Zentrum München, das vom Bundesministerium für Bildung und Forschung sowie vom Freistaat Bayern gefördert wird.
KORA-Age wurde vom Bundesministerium für Bildung und Forschung im Rahmen des Förderprogramms „Gesundheit im Alter" gefördert (FKZ 01ET0713, 01ET1003A und 01ET1003C).

\section{Einhaltung ethischer Richtlinien}

Interessenkonflikt. R. Strobl, W. Maier, A. Mielck, J. Fuchs, A. Richter-Kornweitz, J. Gostomzyk und E. Grill geben an, dass kein Interessenkonflikt besteht.

Dieser Beitrag beinhaltet keine Studien an Menschen oder Tieren.

\section{Literatur}

1. Yen IH, Michael YL, Perdue L (2009) Neighborhood environment in studies of health of older adults: a systematic review. Am J Prev Med 37:455-463

2. Richter A, Wächter M (2009) Zum Zusammenhang von Nachbarschaft und Gesundheit. In: (BZgA) BfgA (Hrsg) Forschung und Praxis in der Gesundheitsförderung, Bd 36. BZgA, Köln

3. Oswald F, Wahl HW, Schilling $O$ et al (2007) Relationships between housing and healthy aging in very old age. Gerontologist 47:96-107

4. Hollbach-Grömig B, Seidel-Schulze A (2007) Seniorenbezogene Gesundheitsförderung und Prävention auf kommunaler Ebene - eine Bestandsaufnahme. In: (BZgA) BfgA (Hrsg) Forschung und Praxis in der Gesundheitsförderung, Bd 33. BZgA, Köln

5. Diez Roux AV, Mair C (2010) Neighborhoods and health. Ann NY Acad Sci 1186:125-145

6. WHO (1986) Ottawa-Charta zur Gesundheitsförderung. WHO, Ottawa

7. Krueger RA, Casey MA (2009) Focus groups: a practical guide for applied reseach. SAGE, California

8. Mayring P (2010) Qualitative Inhaltsanalyse. Beltz, Basel

9. Kawachi I, Berkman LF (2003) Neighborhoods and health. Oxford University Press, New York

10. Statistisches Bundesamt (2009) Bevölkerung Deutschlands bis 2060. Ergebnisse der 12. koordinierten Bevölkerungsvorausberechnung. Statistisches Bundesamt, Wiesbaden

11. World Health Organization (2002) Active aging: a policy framework. WHO, Geneva

12. Bundesministerium für Gesundheit (Hrsg) (2008) Das Robert Koch-Institut - das Public Health Institut für Deutschland, RKI 2010. BMG, Berlin 